antibodies were not seen with proteins of this structure, making it feasible to pursue more extensive preclinical and clinical testing.

The half-life in rats of the IL-12/IL-18 bispecific antibody was similar to that of the original IgG1, consistent with its containing an intact constant region. The protein showed bivalent binding to both cytokines, binding each with an affinity similar to that of the original monospecific antibody. In a severe combined immunodeficient mouse model engrafted with human peripheral blood mononuclear cells, the IL-12/IL-18 bispecific antibody was as effective at inhibiting IFN- $\gamma$ production induced by Staphylococcus aureus dried cells as was a combination of the two original anti-IL-12 and anti-IL-18 monospecific IgG1s. A second IL$1 \alpha /$ IL- $1 \beta$ bispecific molecule was more effective than a single antibody in recognizing both IL- $1 \alpha$ and IL- $1 \beta$ in a mouse collagen-induced arthritis model of rheumatoid arthritis.

The bispecific antibody format described by the authors shares many properties with a singlechain bispecific antibody described by Natsume et al..$^{7}$ (Fig. 1f). In this molecule, tandem singlechain Fvs specific for tumor-associated glycoprotein (TAG)-72 and MUC1 mucin were linked to the Fc of a human IgG1 heavy chain. The bispecific antibody was effectively produced in Chinese hamster ovary cells, retained the ability to recognize both TAG-72 and MUC1 and-in the presence of the appropriate carbohydrate on the constant region-triggered antibody-dependent cellular cytotoxicity in cells expressing TAG-72 or MUC1.
The report by Wu et al. provides convincing evidence that it will now be possible to produce bispecific antibodies that can simultaneously bind and neutralize two soluble proteins. However, the question of their immunogenicity remains and can only be answered by examining the immune response in treated patients. It is also unclear whether DVD-Igs will be capable of linking two different populations of cells. Early on it was hypothesized that bispecific antibodies recognizing two cell surface proteins on two different cell types could bring the two populations into close proximity. Such antibodies might be used to redirect cytotoxic immune cells to destroy pathogenic target cells, including tumors or virally infected cells, or to bring two cell populations together for activation.

Wu et al. have shown that a bispecific protein recognizing both $\mathrm{CD} 3$ and $\mathrm{CD} 20$ can be made, but it remains to be determined whether it can be used to target CD3-expressing T cells to CD20-expressing B cells. However, the expression levels and homogeneity of the product made in the isolated cell lines suggest that it will soon be possible to answer these important questions in preclinical experiments.

1. Wu, C.Y.H., Grinnell, C. \& Bryant, S. Nat. Biotechnol. 25, 1290-1297 (2007).

2. Milstein, C. \& Cuello, A.C. Nature 305, 537-540 (1983).

3. Carter, P. J. Immunol. Methods 248, 7-15 (2001).

4. Holliger, P., Prospero, T. \& Winter, G. Proc. Natl. Acad. Sci. USA 90, 6444-6448 (1993).

5. Pack, P., Muller, K., Zahn, R. \& Pluckthun, A. J. Mol. Biol. 246, 28-34 (1995)

6. Pack, P. \& Pluckthun, A. Biochemistry 31, 1579-1584 (1992).

7. Natsume, A. et al. J. Biochem. 140, 359-368 (2006).

\title{
Fluorescence nanoscopy goes
} multicolor

\author{
Andreas Schönle \& Stefan W Hell
}

\section{Photoswitchable fluorescent labels allow simultaneous optical imaging of multiple target complexes inside fixed cells with nanometer spatial resolution.}

Shades of gray might readily capture the scene, but without color we may miss the essential. This basic fact holds not only for appreciating the sight of turning leaves in the fall but also for unraveling the distribution of

Andreas Schönle and Stefan W. Hell are in the Department of NanoBiophotonics, Max Planck Institute for Biophysical Chemistry, 37070 Göttingen, Germany.

e-mail: aschoen@gwdg.de or shell@gwdg.de. biomolecules in a cell using fluorescence. In a recent report in Science, Bates et al. ${ }^{1}$ introduced a powerful technique of multi-color labeling in fluorescence nanoscopy.

Imaging molecules represented by different hues with a microscope reveals their spatial correlation. But standard microscopy techniques are limited in resolution by diffraction, implying that densely packed biomolecules cannot be located more precisely than within $\sim 200 \mathrm{~nm}$. Although tight prox- imities $(<5 \mathrm{~nm})$ of molecular pairs can be sensed by Förster energy transfer (FRET), spatial correlations in the 5- to 200 -nm range have remained largely uncharted.

In the last decade, however, physical concepts have emerged that overcome the diffraction limit by exploiting the spectral properties of fluorophores ${ }^{2}$. Stimulated emission depletion (STED) microscopy and the photoswitching-based single-molecule nanoscopies now routinely provide resolution of 20-50 nm. Therefore, an obvious solution is to endow these techniques with multiple color channels. But as each of these methods brings new constraints, this is easier said than done. Hence, it is not surprising that dual-color fluorescence nanoscopy by STED $^{3}$ and single-molecule photoswitching ${ }^{4}$ has been demonstrated only very recently.

Extending on their development of stochastic reconstruction microscopy $(\mathrm{STORM})^{5}$ with pairs of cyanine dyes, Bates et al. have now taken a bolder step: $25-\mathrm{nm}$ far-field fluorescence microscopy resolution with triple-color operation. Moreover, they describe an astonishingly modular system of coupled photoswitchable cyanine dyes with the potential of accommodating nine or even more color channels. All of a sudden, recording a whole gamut of labeled biomolecules with nanoscale resolution has come within reach.

How does it work? STORM and the other closely related nanoscopy techniques based on activation and deactivation of isolated molecules ${ }^{6-8}$ use molecular switching in a powerful way. Whereas the ensemble switching concepts ${ }^{9}$, such as STED, produce tiny focal spots of activated molecules by deactivating those right outside those tiny spots, these techniques switch on individual photoactivatable molecules so sparsely that they can be easily discerned on a camera. Exciting the activated molecules yields a blob of $m$ detected photons on the camera, allowing calculation of the spatial coordinate of the label with $\sim \lambda /(2 \sqrt{m})$ precision. The coordinate is then tick-marked in the image, and the registered label goes back to the deactivated state. This on-off cycle is repeated until the tick-marks form an image.

Multiple colors can be accommodated in this approach by using labels that differ in either their activation or their fluorescence excitation or their emission $\operatorname{spectra}^{4}$ (Fig. 1). However, requirement for both an activation and an excitation beam increases complexity because one of the beams may undesirably do the job of the other. Additionally, some of these photoswitchable dyes require specific chemical environments to switch reliably. 
a

a
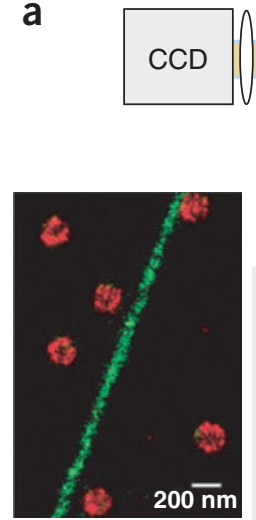
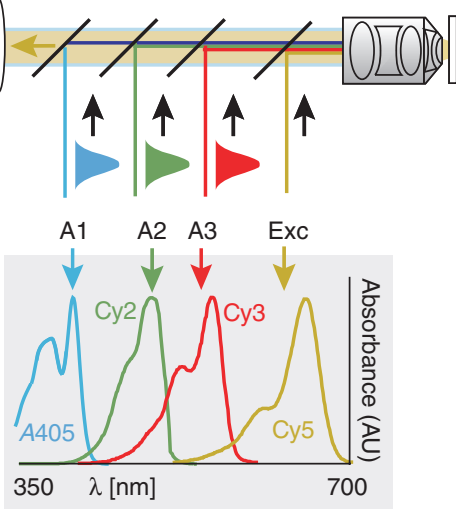

b

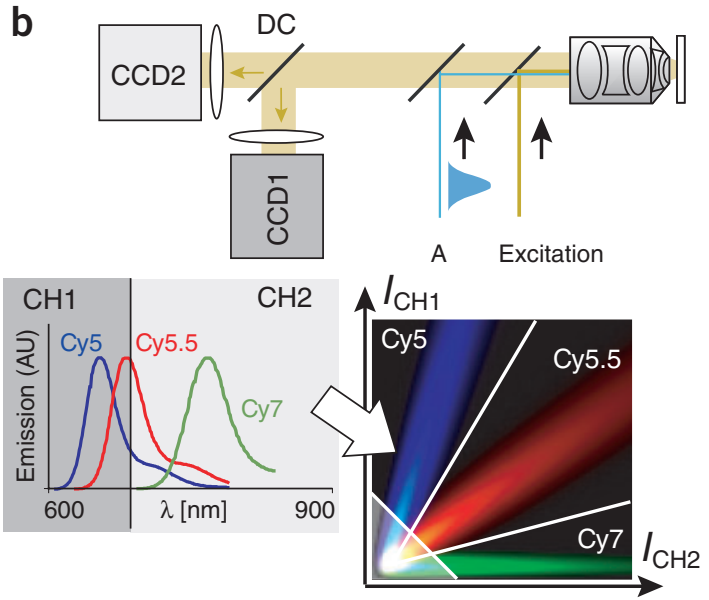

Figure 1 Complementary strategies for multicolor far-field fluorescence nanoscopy based on photoswitching of individual fluorescence emitters. The setup is based on an epifluorescence microscope with different light sources and an optional second detection color channel. (a) Bates et al. use coupled pairs of 'activator' and switchable 'fluorescence reporter' molecules. Imaging was performed using Alexa 405 (A405), Cy2 and Cy3 as activators and Cy5 or Alexa 647 as a reporter. Whereas the fluorescence of the reporter is elicited by a single excitation beam (Exc), selectivity is gained by exciting each activator consecutively with laser pulses of specific wavelengths (A1-A3). Lower left panel displays a STORM micrograph showing clathrin coated pits (red, Cy3-Alexa 647) and microtubules (green, Cy2-Alexa 647) with an effective resolution of $25 \mathrm{~nm}$. AU, arbitrary units. (b) An alternative method is to discriminate fluorescent labels by (small) shifts in their emission spectra. The fluorescence of the switchable molecule is split into two color channels. Because individual emitters are recorded separately, the intensity ratio between the channels is sufficient to identify each emitter. In a two-dimensional grid spanned by the number of detected photons in each channel $\left(I_{\mathrm{ch} 1}, I_{\mathrm{ch} 2}\right)$, a fluorescence event will fall onto the line representing its characteristic ratio $I_{\text {ch } 1} / I_{\text {ch2 }}$. Shot-noise and background blur these distributions (lower right), but if bright enough, each single-molecule detection event can still be assigned reliably to one of the species in question. The data were simulated using the emission spectra of Cy5, Cy5.5 and Cy 7 , assuming an average signal of 240 (Cy5, Cy5.5) and 40 (Cy7) photons per event and background noise of 5 photons per channel per event. The maximum intensity displayed for each channel is 100 photons. When the event threshold is chosen at $>30$ photons, as indicated by the white bar, cross-talk amounts to $<1 \%$ for all species, highlighting the potential of this approach.

The authors bypassed these problems with pairs of cyanine dyes consisting of an 'activator' and a 'reporter'. Excited by the activation beam, the 'activator' switches on the 'reporter' whose fluorescence is read out. Thus they developed a modular system consisting of three activators and three reporters all featuring different excitation wavelengths, allowing an impressive nine distinct combinations. An inherent advantage of this approach is that all fluorophores are similar in structure and hence operate under the same chemical environment.

We think this is just the tip of the iceberg. Unlike in bulk measurements, where the number of detection channels has to match or exceed that of the label species, in singlemolecule recordings, two color channels are enough to discriminate between several emission spectra. Because all photons can be assigned to the same emitter, the ratio of the counts in each channel suffices to identify changes in the spectrum and hence the type of label. (By contrast, in the bulk, arbitrary intensity ratios are possible, depending on the local ratio of label concentrations.) Thus, at a given number of detected photons, individually activated labels can be swiftly identified. Figure $\mathbf{1 b}$ shows a simulation assuming 240 detected photons per molecule, which is 25 times lower than the number of photons reported by Bates et al.. Nonetheless, even with five counts of background noise, the three molecular species are clearly distinguished.

Spectral analyses of this kind are well established in single-molecule spectroscopy but are not easily translated to imaging. This is because the limited resolution inevitably leads to measurements in bulk. Photoswitching of individual, sparsely distributed molecules now allows the assignment of the measured photons to individual emitters even in densely labeled samples. This, by the way, holds not only for singlemolecule switching but also for the other nanoscopy approaches, such as STED, if the detection volume is reduced such that only a single molecule is in that volume at a time. In conclusion, sometimes seeing less is seeing more.

The opportunities that arise from the isolation of single emitters in nanoscopy go far beyond identification of densely packed molecules by color. In fact, the whole range of parameters, including fluorescence lifetime, anisotropy and FRET efficiency, becomes accessible regardless of the local concentration of molecules ${ }^{10}$. Multiple colors are not the end of the story.

1. Bates, M., Huang, B., Dempsey, G.P. \& Zhuang, X. Science 317, 1749-1753 (2007).

2. Hell, S.W. Science 316, 1153-1158 (2007).

3. Donnert, G. et al. Biophys. J. 92, L67-L69 (2007).

4. Bock, H. et al. Appl. Phys. B 88, 161-165 (2007).

5. Rust, M.J., Bates, M. \& Zhuang, X. Nat. Methods 3, 793-796 (2006).

6. Betzig, E. et al. Science 313, 1642-1645 (2006).

7. Hess, S.T., Girirajan, T.P.K. \& Mason, M.D. Biophys. J. 91, 4258-4272 (2006).

8. Egner, A. et al. Biophys. J. 93, 3285-3290 (2007).

9. Hell, S.W. Nat. Biotechnol. 21, 1347-1355 (2003).

10. Eggeling, C. et al. Micr. Res. Tech. doi:10.1002/ jemt.20505 (27 July 2007). 